\title{
On nontrivial solutions of nonlinear Schrödinger equations with sign-changing potential
}

\author{
Wei Chen ${ }^{1,2}$, Yue $\mathrm{Wu}^{1 *}$ (D) and Seongtae Jhang ${ }^{2}$
}

\section{"Correspondence:}

wuyue@lyu.edu.cn

'School of Mathematics and

Statistics, Linyi University, 276000

Linyi, China

Full list of author information is

available at the end of the article

\section{Springer}

\begin{abstract}
In this paper, we consider the superlinear Schrödinger equation with bounded potential well. The potential here is allowed to be sign-changing. Without assuming the Ambrosetti-Rabinowitz-type condition, we prove the existence of a nontrivial solution and multiplicity results.
\end{abstract}

Keywords: Schrödinger equation; Superlinear; Potential well; Variational methods

\section{Introduction and main results}

This paper is concerned with the existence and multiplicity of nontrivial solutions for the superlinear Schrödinger equation of the form

$$
\left\{\begin{array}{l}
-\Delta u+V(x) u=f(x, u), \\
u \in H^{1}\left(\mathbb{R}^{N}\right), \quad N \geq 3 .
\end{array}\right.
$$

With the aid of variational methods, problems of the form (1.1) have been extensively studied in the past decades. There are many works adopting various assumptions on $V$ and $f$; see, for example, [1-13] and references therein.

Motivated by the above works, in this paper, we consider equation (1.1) with a signchanging potential well. For the potential $V$, we assume:

(V) $V \in C\left(\mathbb{R}^{N}\right), V(x)<V_{\infty}:=\lim _{|x| \rightarrow \infty} V(x)<\infty, 0 \notin \sigma(-\Delta+V)$, the spectrum of $-\Delta+V$.

Remark 1.1 Define the nondecreasing sequence of minimax values by

$$
\lambda_{n}=\lim _{S \in \mathcal{S}_{n}} \sup _{u \in S \backslash\{0\}} \frac{\int_{\mathbb{R}^{N}}\left(|\nabla u|^{2}+V(x) u^{2}\right) \mathrm{d} x}{\int_{\mathbb{R}^{N}} u^{2} \mathrm{~d} x}, \quad n \in \mathbb{N},
$$

where $\mathcal{S}_{n}$ is a family of $n$-dimensional subspaces of $C_{0}^{\infty}\left(\mathbb{R}^{N}\right)$. We can see that $\sigma_{\text {ess }}(-\Delta+$ $V) \in\left(V_{\infty}, \infty\right)$ by $(\mathrm{V}), \lambda_{\infty}:=\lim _{k \rightarrow \infty} \lambda_{n}=\inf \sigma_{\mathrm{ess}}(-\Delta+V)<\infty$, and $\lambda_{n} \in \sigma_{p p}(-\Delta+V)$

(c) The Author(s) 2021. This article is licensed under a Creative Commons Attribution 4.0 International License, which permits use, sharing, adaptation, distribution and reproduction in any medium or format, as long as you give appropriate credit to the original author(s) and the source, provide a link to the Creative Commons licence, and indicate if changes were made. The images or other third party material in this article are included in the article's Creative Commons licence, unless indicated otherwise in a credit line to the material. If material is not included in the article's Creative Commons licence and your intended use is not permitted by statutory regulation or exceeds the permitted use, you will need to obtain permission directly from the copyright holder. To view a copy of this licence, visit http://creativecommons.org/licenses/by/4.0/. 
whenever $\lambda_{n}<\lambda_{\infty}$, where $\sigma_{\text {ess }}(-\Delta+V)$ denotes the essential spectrum of $-\Delta+V$, and $\sigma_{p p}(-\Delta+V)$ denotes the pure point spectrum of $-\Delta+V$ (see $[14,15]$ for details).

Besides (V), in [11, 12], it is also assumed that $\inf V>0$, so that $\lambda_{1}>0$. Then the energy functional with respect to problem (1.1) has mountain pass geometry. In this work, we are interested in the case where the Schrödinger operator $-\Delta+V$ possesses a nontrivial negative space, which leads to more difficulty in verifying the compactness conditions. To the best of our knowledge, there are not many results in this case.

In this paper, we do not assume any compactness conditions on the potential function $V$. It is well known that the main difficulty in studying (1.1) in $\mathbb{R}^{N}$ is the lack of compactness. This difficulty can be avoided for (1.1) in bounded domains or if the potential function $V$ possesses some compactness conditions. For example, if $\lim _{|x| \rightarrow \infty} V(x)=\infty$ or $u$ is radially symmetric, we can get some compactness embedding, and then the Palias-Smale condition can be proved. We refer to [16] in this direction.

Denote $F(x, t):=\int_{0}^{t} f(x, s) \mathrm{d} s, 2^{*}:=\frac{2 N}{N-2}$, and $p^{\prime}:=\frac{p}{p-1}$, the conjugate exponent of $p$. We make the following assumptions on the nonlinearity $f$.

$\left(f_{1}\right) f \in C^{1}\left(\mathbb{R}^{N} \times \mathbb{R}\right)$, and there exist constants $p \in\left(2,2^{*}\right)$ and $c>0$ such that

$$
|f(x, t)| \leq c\left(1+|t|^{p-1}\right)
$$

for $x \in \mathbb{R}^{N}$ and $t \in \mathbb{R}$

$\left(f_{2}\right) f(x, t)=o(t)$ as $t \rightarrow 0$ uniformly in $x \in \mathbb{R}^{N}$.

$\left(f_{3}\right) F(x, t) / t^{2} \rightarrow \infty$ as $|t| \rightarrow \infty$ uniformly in $x \in \mathbb{R}^{N}$.

$\left(f_{4}\right) \lim _{|x| \rightarrow \infty} \sup _{|t| \leq l} \frac{|f(x, t)|}{|t|}=0$ for every $l>0$.

$\left(f_{5}\right)$ There exist $a, b>0$ and $\alpha \in\left(0, \alpha_{*}\right)$ such that

$$
0<\left(2+\frac{1}{a|t|^{\alpha}+b}\right) F(x, t) \leq t f(x, t)
$$

for $x \in \mathbb{R}^{N}$ and $t \neq 0$, where $\alpha_{*}=\min \left\{p^{\prime},\left(2^{*}-1\right) p^{\prime}-2^{*}\right\}$.

Then we have the following two results.

Theorem 1.2 Under assumptions $(\mathrm{V})$ and $\left(f_{1}\right)-\left(f_{5}\right)$, problem $(1.1)$ possesses at least one nontrivial solution.

Theorem 1.3 Under assumptions of Theorem 1.2, if $f(x, t)$ is odd in $t$, then problem (1.1) possesses infinitely many solutions.

Remark 1.4 To produce critical points of the variational functional of (1.1), we will eventually encounter the compactness problem. For this issue, we introduced assumption $\left(f_{4}\right)$. It is easy to see that if $a: \mathbb{R}^{N} \rightarrow \mathbb{R}$ is continuous, $\lim _{|x| \rightarrow \infty} a(x)=0$, and $p \in\left(2,2^{*}\right)$, then

$$
f(x, t)=a(x)|t|^{p-2} t
$$

satisfies $\left(f_{1}\right)-\left(f_{5}\right)$.

Remark 1.5 Most papers concerned with the superlinear Schrödinger equations involve the following classical condition of Ambrosetti and Rabinowitz: 
(AR) There exists $\mu>2$ such that $0<\mu F(x, t) \leq t f(x, t)$ for all $x \in \mathbb{R}^{N}$ and $t \neq 0$.

Condition (AR) plays a crucial role in proving the boundedness of Palias-Smale or Cerami sequences. Instead, we introduce a new condition $\left(f_{5}\right)$, and we will illustrate a general technique to establish the boundedness of Cerami sequences. It is well known that many superlinear nonlinearities such as

$$
f(x, t)=t \ln (1+|t|)
$$

do not satisfy condition (AR). Note that $\frac{1}{a|t|^{\alpha}+b} \rightarrow 0$ as $|t| \rightarrow \infty$, which indicates that $\left(f_{5}\right)$ is somewhat weaker than (AR). Note also that $\left(2^{*}-1\right) p^{\prime}-2^{*}>0$ whenever $p<2^{*}$. So the parameter $\alpha \in\left(0, \alpha_{*}\right)$ is available. It is also worth pointing out that $\left(f_{5}\right)$ is not a superlinear condition. Indeed, there are asymptotically linear functions satisfying $\left(f_{5}\right)$.

\section{Preliminaries}

We denote by $E:=H^{1}\left(\mathbb{R}^{N}\right)$ the usual Sobolev space. Define the functional $\Phi: E \rightarrow \mathbb{R}$ by

$$
\Phi(u)=\frac{1}{2} \int_{\mathbb{R}^{N}}\left(|\nabla u|^{2}+V(x) u^{2}\right) \mathrm{d} x-\int_{\mathbb{R}^{N}} F(x, u) \mathrm{d} x .
$$

Our assumptions on $V$ and $f$ stated above imply that the Schrödnger operator $-\Delta+V$ is selfadjoint and semibounded in $L^{2}\left(\mathbb{R}^{N}\right)$ and $\Phi \in C^{1}(E, \mathbb{R})$. A direct computation gives that, for all $u, v \in E$,

$$
\left\langle\Phi^{\prime}(u), v\right\rangle=\int_{\mathbb{R}^{N}}(\nabla u \nabla v+V(x) u v) \mathrm{d} x-\int_{\mathbb{R}^{N}} f(x, u) v \mathrm{~d} x .
$$

It is well known that the critical points of $\Phi$ are solutions of problem (1.1).

By (V) 0 is not an eigenvalue of $-\Delta+V$. If $\lambda_{1}>0$, we easily see that $\Phi$ has the mountain pass geometry. This case is simple, and we omit it here. In view of Remark 1.1, we arrange the eigenvalues (counted with multiplicity) of $-\Delta+V$ as

$$
-\infty<\lambda_{1} \leq \lambda_{2} \leq \cdots \leq \lambda_{\ell}<0<\lambda_{\ell+1} \leq \cdots<\lambda_{\infty}
$$

and denote by $e_{j}$ the corresponding eigenfunction of $\lambda_{j}$. Let $E^{-}=\operatorname{span}\left\{e_{1}, \ldots, e_{\ell}\right\}$ and $E^{+}=$ $\left(E^{-}\right)^{\perp}$. From $(\mathrm{V})$ we deduce that $E=E^{-} \oplus E^{+}$, where $E^{-}$and $E^{+}$are the negative and positive eigenspaces of the operator $-\Delta+V$, and that $\operatorname{dim} E^{-}<\infty$. For $u, v \in E$, define

$$
(u, v)=\int_{\mathbb{R}^{N}}\left(\nabla u^{+} \nabla v^{+}+V(x) u^{+} v^{+}\right) \mathrm{d} x-\int_{\mathbb{R}^{N}}\left(\nabla u^{-} \nabla v^{-}+V(x) u^{-} v^{-}\right) \mathrm{d} x,
$$

where $u=u^{-}+u^{+}$with $u^{-} \in E^{-}$and $u^{+} \in E^{+}$. Then $(\cdot, \cdot)$ is an inner product on $E$. Therefore $E$ is a Hilbert space with the norm $\|\cdot\|:=\sqrt{(\cdot, \cdot)}$. We easily see that

$$
\Phi(u)=\frac{1}{2}\left\|u^{+}\right\|^{2}-\frac{1}{2}\left\|u^{-}\right\|^{2}-\int_{\mathbb{R}^{N}} F(x, u) \mathrm{d} x
$$

and

$$
\left\langle\Phi^{\prime}(u), v\right\rangle=\left(u^{+}, v^{+}\right)-\left(u^{-}, v^{-}\right)-\int_{\mathbb{R}^{N}} f(x, u) v \mathrm{~d} x .
$$


For any $s \in\left[2,2^{*}\right]$, the imbedding $E \hookrightarrow L^{s}\left(\mathbb{R}^{N}\right)$ is continuous. Consequently, there exists a constant $\tau_{s}>0$ such that

$$
|u|_{s} \leq \tau_{s}\|u\|, \quad \forall u \in E,
$$

where $|\cdot|_{s}$ denotes the $L^{s}$ norm.

We next recall some abstract critical point theorems, which will be used in the proofs of our main results.

Definition 2.1 Let $E$ be a Banach space, and let $\Phi \in C^{1}(E, \mathbb{R})$. Given $c \in \mathbb{R}$, a sequence $\left\{u_{n}\right\} \subset E$ is called a Cerami sequence of $\Phi$ at level $c$ (shortly, a $(C)_{c}$ sequence) if

$$
\Phi\left(u_{n}\right) \rightarrow c, \quad\left(1+\left\|u_{n}\right\|\right)\left\|\Phi^{\prime}\left(u_{n}\right)\right\| \rightarrow 0
$$

We say that $\Phi$ satisfies the Cerami condition at level $c$ (shortly, condition $(C)_{c}$ ) if every $(C)_{c}$ sequence of $\Phi$ contains a convergent subsequence. If $\Phi$ satisfies condition $(C)_{c}$ for every $c \in \mathbb{R}$, then we say that $\Phi$ satisfies the Cerami condition (shortly, condition (C)).

Obviously, condition $(\mathrm{C})$ is weaker than the Palais-Smale condition. However, as was shown in [17], the deformation theorem is still valid under the Cerami condition. Thus we have the following theorems.

Theorem 2.2 (Linking theorem [18]) Let $E=E^{-} \oplus E^{+}$be a Banach space with $\operatorname{dim} E^{-}<\infty$. Let $R>r>0$, and let $\phi \in E^{+} \backslash\{0\}$. Define

$$
M:=\left\{u=u^{-}+\lambda \phi \mid u^{-} \in E^{-},\|u\| \leq R, \lambda \geq 0\right\}, \quad N:=\left\{u \in E^{+} \mid\|u\|=r\right\} .
$$

If $\Phi \in C^{1}(E, \mathbb{R})$ satisfies condition $(C)$ and

$$
\inf _{N} \Phi>\max _{\partial M} \Phi
$$

then $\Phi$ has a nontrivial critical point.

For the proofs of Theorems 1.2-1.3, we will use the following fountain theorem, which is a generalization of the classical fountain theorem of Bartsch [19] (see also [10]). For $k \in \mathbb{N}$, let

$$
Y_{k}=\operatorname{span}\left\{e_{1}, \ldots e_{k}\right\}, \quad Z_{k}=Y_{k}^{\perp} .
$$

Theorem 2.3 (Fountain theorem [20]) Suppose that the functional $\Phi \in C^{1}(E, \mathbb{R})$ is even and satisfies condition (C). Suppose that for every $k \geq k_{0}$ for some constant $k_{0}>0$, there exist $\rho_{k}>r_{k}>0$ such that

$\left(\mathrm{A}_{1}\right) b_{k}=\inf _{u \in Z_{k},\|u\|=r_{k}} \Phi(u) \rightarrow \infty$ as $k \rightarrow \infty$, and

$\left(\mathrm{A}_{2}\right) a_{k}=\max _{u \in Y_{k},\|u\|=\rho_{k}} \Phi(u) \leq 0$.

Then $\Phi$ has a sequence of critical points $\left\{u_{k}\right\}$ such that $\Phi\left(u_{k}\right) \rightarrow \infty$. 


\section{Proof of main results}

Lemma 3.1 Suppose that $(\mathrm{V}),\left(f_{1}\right)$, and $\left(f_{2}\right)$ are satisfied. Then there exists $r>0$ such that $\inf \Phi\left(\partial B_{r}(\mathbf{0}) \cap E^{+}\right)>0$.

Proof It follows from $\left(f_{1}\right)$ and $\left(f_{2}\right)$ that, for given $\varepsilon>0$, there is a constant $C_{\varepsilon}>0$ such that

$$
|F(x, t)| \leq \varepsilon|t|^{2}+C_{\varepsilon}|t|^{p}
$$

and

$$
|f(x, t)| \leq \varepsilon|t|+C_{\varepsilon}|t|^{p-1} .
$$

For $u \in E^{+}$, we have

$$
\begin{aligned}
\Phi(u) & =\frac{1}{2}\|u\|^{2}-\int_{\mathbb{R}^{N}} F(x, u) \mathrm{d} x \\
& \geq \frac{1}{2}\|u\|^{2}-\varepsilon|u|_{2}^{2}-C_{\varepsilon}|u|_{p}^{p} \\
& \geq\left(\frac{1}{2}-\varepsilon \tau_{2}\right)\|u\|^{2}-\tau_{p} C_{\varepsilon}\|u\|^{p},
\end{aligned}
$$

where $\tau_{2}$ and $\tau_{p}$ are constants in (2.4). Let $\varepsilon=\frac{1}{4 \tau_{2}}$. Since $p>2$, we can fix some $r$ small enough such that

$$
\inf _{u \in E^{+},\|u\|=r} \Phi(u)>0
$$

The proof is completed.

Lemma 3.2 Suppose that $(\mathrm{V})$ and $\left(f_{1}\right)-\left(f_{3}\right)$ are satisfied. Then, for any nontrivial finitedimensional subspace $W$ of $E^{+}$, there exists $R>r$ such that $\Phi \leq 0$ in $\left(E^{-} \oplus W\right) \backslash B_{R}(0)$, where $r>0$ is the constant given by Lemma 3.1.

Proof This lemma is a corollary of [13, Lemma 2.5]. We omit the proof.

Lemma 3.3 Suppose that $(\mathrm{V}),\left(f_{1}\right)-\left(f_{3}\right)$, and $\left(f_{5}\right)$ are satisfied and $c \in \mathbb{R}$. Then any $(C)_{c}$ sequence of $\Phi$ is bounded.

Proof It follows from $\left(f_{5}\right)$ that, for all $t \neq 0$ and $x \in \mathbb{R}^{N}$,

$$
t f(x, t)-2 F(x, t) \geq \frac{1}{2 a|t|^{\alpha}+2 b+1} t f(x, t)>0 .
$$

Let $\left\{u_{n}\right\}$ be a $(C)_{c}$ sequence of $\Phi$, that is, a sequence satisfying (2.5). Set $\Pi_{n}:=\{x \in$ $\left.\mathbb{R}^{N}|| u_{n}(x) \mid<1\right\}$ and $\Pi_{n}^{c}:=\mathbb{R}^{N} \backslash \Pi_{n}$. Then there are constants $c_{1}, c_{2}>0$ such that

$$
2 a\left|u_{n}\right|^{\alpha}+2 b+1 \leq 1 / c_{1}, \quad \forall x \in \Pi_{n},
$$


and

$$
2 a\left|u_{n}\right|^{\alpha}+2 b+1 \leq\left|u_{n}\right|^{\alpha} / c_{2}, \quad \forall x \in \Pi_{n}^{c} .
$$

For $n$ sufficient large, it follows that

$$
\begin{aligned}
D & \geq 2 \Phi\left(u_{n}\right)-\left\langle\Phi^{\prime}\left(u_{n}\right), u_{n}\right\rangle \\
& =\int_{\mathbb{R}^{N}}\left(u_{n} f\left(x, u_{n}\right)-2 F\left(x, u_{n}\right)\right) \mathrm{d} x \\
& \geq \int_{\mathbb{R}^{N}} \frac{u_{n} f\left(x, u_{n}\right)}{2 a\left|u_{n}\right|^{\alpha}+2 b+1} \mathrm{~d} x \\
& \geq c_{1} \int_{\Pi_{n}} u_{n} f\left(x, u_{n}\right) \mathrm{d} x+c_{2} \int_{\Pi_{n}^{c}}\left|u_{n}\right|^{-\alpha} u_{n} f\left(x, u_{n}\right) \mathrm{d} x
\end{aligned}
$$

for some constant $D>0$.

Note that $\alpha<\left(2^{*}-1\right) p^{\prime}-2^{*}$ by $\left(f_{5}\right)$. We have

$$
\frac{1}{p^{\prime}}<\frac{2^{*}}{2^{*}-1} \frac{1}{p^{\prime}}<\frac{2^{*}}{2^{*}+\alpha} \text { and } \quad \frac{2}{2+\alpha}<\frac{2^{*}}{2^{*}+\alpha} .
$$

Then we can choose a constant $r \in(0,1)$ such that

$$
\max \left\{\frac{2^{*}}{2^{*}-1} \frac{1}{p^{\prime}}, \frac{2}{2+\alpha}\right\}<r<\frac{2^{*}}{2^{*}+\alpha}
$$

Let $s:=r /(1-r)>0$. Then $\frac{1}{r}+\frac{1}{-s}=1$. By (3.3) and the inverse Hölder inequality we have

$$
\begin{aligned}
D & \geq c_{1} \int_{\Pi_{n}} u_{n} f\left(x, u_{n}\right) \mathrm{d} x+c_{2}\left(\int_{\Pi_{n}^{c}}\left(u_{n} f\left(x, u_{n}\right)\right)^{r} \mathrm{~d} x\right)^{1 / r}\left(\int_{\Pi_{n}^{c}}\left|u_{n}\right|^{\alpha s} \mathrm{~d} x\right)^{1 /(-s)} \\
& \geq c_{1} \int_{\Pi_{n}} u_{n} f\left(x, u_{n}\right) \mathrm{d} x+c_{2} \frac{\left(\int_{\Pi_{n}^{c}}\left(u_{n} f\left(x, u_{n}\right)\right)^{r} \mathrm{~d} x\right)^{1 / r}}{\left|u_{n}\right|_{\alpha s}^{\alpha}} .
\end{aligned}
$$

By $\left(f_{1}\right)$ and $\left(f_{2}\right)$ we have

$$
\begin{aligned}
& |f(x, u)|^{p^{\prime} r} \leq\left(c_{3}|u|^{(p-1)\left(p^{\prime}-1\right)}|f(x, u)|\right)^{r}=c_{4}(u f(x, u))^{r}, \quad \forall|u| \geq 1, \\
& |f(x, u)|^{2} \leq c_{5}|u||f(x, u)|=c_{5} u f(x, u), \quad \forall|u|<1 .
\end{aligned}
$$

Therefore by (3.5) we have

$$
\begin{aligned}
& \left(\int_{\Pi_{n}^{c}}\left|f\left(x, u_{n}\right)\right|^{p^{\prime} r} \mathrm{~d} x\right)^{1 / p^{\prime} r} \leq c_{6}\left|u_{n}\right|_{\alpha s}^{\alpha / p^{\prime}}, \\
& \left(\int_{\Pi_{n}}\left|f\left(x, u_{n}\right)\right|^{2} \mathrm{~d} x\right)^{1 / 2} \leq c_{7} .
\end{aligned}
$$

In view of (3.4), we easily check that $p^{\prime} r>1$ and $\left(p^{\prime} r\right)^{\prime}, \alpha s \in\left[2,2^{*}\right]$, where $\left(p^{\prime} r\right)^{\prime}:=$ $p^{\prime} r /\left(p^{\prime} r-1\right)$. Consequently, it follows from (3.6) and (3.7), Hölder's inequality, and 
Sobolev's inequality that, for $n$ large enough and some constants $c_{8}, c_{9}>0$,

$$
\begin{aligned}
\left\|u_{n}^{+}\right\|^{2} & =\left\langle\Phi^{\prime}\left(u_{n}\right), u_{n}^{+}\right\rangle+\int_{\mathbb{R}^{N}} f\left(x, u_{n}\right) u_{n}^{+} \mathrm{d} x \\
& \leq\left\|u_{n}^{+}\right\|+\left(\int_{\Pi_{n}}\left|f\left(x, u_{n}\right)\right|^{2} \mathrm{~d} x\right)^{1 / 2}\left|u_{n}^{+}\right|_{2}+\left(\int_{\Pi_{n}^{c}}\left|f\left(x, u_{n}\right)\right|^{p^{\prime} r} \mathrm{~d} x\right)^{1 / p^{\prime} r}\left|u_{n}^{+}\right|_{\left(p^{\prime} r\right)^{\prime}} \\
& \leq\left\|u_{n}^{+}\right\|+c_{7}\left|u_{n}^{+}\right|_{2}+c_{6}\left|u_{n}\right|_{\alpha s}^{\alpha / p^{\prime}}\left|u_{n}^{+}\right|_{\left(p^{\prime} r\right)^{\prime}} \leq c_{8}\left\|u_{n}^{+}\right\|+c_{9}\left\|u_{n}^{+}\right\|\left\|u_{n}\right\|^{\alpha / p^{\prime}} .
\end{aligned}
$$

Therefore we obtain

$$
\left\|u_{n}^{+}\right\| \leq c_{8}+c_{9}\left\|u_{n}\right\|^{\alpha / p^{\prime}}
$$

and, similarly,

$$
\left\|u_{n}^{-}\right\| \leq c_{8}+c_{9}\left\|u_{n}\right\|^{\alpha / p^{\prime}}
$$

Note that $\alpha<p^{\prime}$. Then we easily verify that $\left\|u_{n}\right\|^{2}=\left\|u_{n}^{-}\right\|^{2}+\left\|u_{n}^{+}\right\|^{2}$ is bounded.

Lemma 3.4 Suppose that (V) and $\left(f_{1}\right)-\left(f_{4}\right)$ are satisfied. Then any bounded $(C)_{c}$ sequence of $\Phi$ contains a convergent subsequence.

Proof Suppose $\left\{u_{n}\right\}$ is a bounded $(C)_{c}$ sequence of $\Phi$. Then, passing to a subsequence, we may assume that $u_{n} \rightarrow u$ in $E$. Since $\operatorname{dim} E^{-}<\infty$, we have $u_{n}^{+} \rightarrow u^{+}$in $E^{+}, u_{n}^{-} \rightarrow u^{-}$in $E^{-}$, and $u_{n}^{+} \rightarrow u^{+}$in $L_{\text {loc }}^{s}\left(\mathbb{R}^{N}\right), s \in\left[2,2^{*}\right)$. To establish the strong convergence, it suffices to prove that

$$
\left\|u_{n}^{+}\right\| \rightarrow\left\|u^{+}\right\|
$$

Since

$$
\left\langle\Phi^{\prime}\left(u_{n}\right), u_{n}^{+}-u^{+}\right\rangle=\left(u_{n}^{+}, u_{n}^{+}-u^{+}\right)-\int_{\mathbb{R}^{N}} f\left(x, u_{n}\right)\left(u_{n}^{+}-u^{+}\right) \mathrm{d} x \rightarrow 0,
$$

we have

$$
\begin{aligned}
0 & \leq \limsup _{n \rightarrow \infty}\left(\left\|u_{n}^{+}\right\|^{2}-\left\|u^{+}\right\|^{2}\right) \\
& =\limsup _{n \rightarrow \infty}\left(u_{n}^{+}, u_{n}^{+}-u^{+}\right)=\limsup _{n \rightarrow \infty} \int_{\mathbb{R}^{N}} f\left(x, u_{n}\right)\left(u_{n}^{+}-u^{+}\right) \mathrm{d} x .
\end{aligned}
$$

Next, let $\varepsilon>0$. For $l \geq 1$, from $\left(f_{1}\right)$ and Hölder's inequality it follows that

$$
\begin{aligned}
\int_{\left|u_{n}\right| \geq l} f\left(x, u_{n}\right)\left(u_{n}^{+}-u^{+}\right) \mathrm{d} x & \leq 2 c \int_{\left|u_{n}\right| \geq l}\left|u_{n}\right|^{p-1}\left|u_{n}^{+}-u^{+}\right| \mathrm{d} x \\
& \leq 2 c l^{p-2^{*}} \int_{\left|u_{n}\right| \geq l}\left|u_{n}\right|^{2^{*}-1}\left|u_{n}^{+}-u^{+}\right| \mathrm{d} x \\
& \leq 2 c l^{p-2^{*}}\left|u_{n}\right|_{2^{*}}^{2^{*}-1}\left|u_{n}^{+}-u^{+}\right|_{2^{*}}
\end{aligned}
$$


Since $p<2^{*}$, we may fix $l$ large enough such that

$$
\int_{\left|u_{n}\right| \geq l} f\left(x, u_{n}\right)\left(u_{n}^{+}-u^{+}\right) \mathrm{d} x \leq \frac{\varepsilon}{3}
$$

for all $n$. Moreover, by $\left(f_{4}\right)$ there exists $L>0$ such that

$$
\int_{\substack{|x| \geq L \\\left|u_{n}\right| \leq l}} f\left(x, u_{n}\right)\left(u_{n}^{+}-u^{+}\right) \mathrm{d} x \leq\left|u_{n}\right|_{2}\left|u_{n}^{+}-u^{+}\right|_{2} \sup _{|t| \leq l,|x| \geq L} \frac{|f(x, t)|}{|t|} \leq \frac{\varepsilon}{3}
$$

for all $n$. Finally, since $u_{n}^{+} \rightarrow u^{+}$in $L^{s}\left(B_{L}(\mathbf{0})\right)$ for $s \in\left[2,2^{*}\right)$, from (3.2) it follows that

$$
\begin{aligned}
\int_{\substack{|x| \leq L \\
\left|u_{n}\right| \leq l}} f\left(x, u_{n}\right)\left(u_{n}^{+}-u^{+}\right) \mathrm{d} x & \leq \int_{\substack{|x| \leq L \\
\left|u_{n}\right| \leq l}}\left|u_{n}\right|\left|u_{n}^{+}-u^{+}\right| \mathrm{d} x+C_{1} \int_{\substack{|x| \leq L \\
\left|u_{n}\right| \leq l}}\left|u_{n}\right|^{p-1}\left|u_{n}^{+}-u^{+}\right| \mathrm{d} x \\
& \leq\left|u_{n}\right|_{2}\left|u_{n}^{+}-u^{+}\right|_{L^{2}\left(B_{L}(\mathbf{0})\right)}+C_{1}\left|u_{n}\right|_{p}^{p-1}\left|u_{n}^{+}-u^{+}\right|_{L^{p}\left(B_{L}(\mathbf{0})\right)} \\
& \leq \frac{\varepsilon}{3}
\end{aligned}
$$

for $n$ large enough. Combining (3.10)-(3.12), we conclude that

$$
\int_{\mathbb{R}^{N}} f\left(x, u_{n}\right)\left(u_{n}^{+}-u^{+}\right) \mathrm{d} x \leq \varepsilon
$$

for $n$ large enough. Since $\varepsilon$ is arbitrary, this, together with (3.9), implies (3.8). The lemma is proved.

Proof of Theorem 1.2 For $u \in E^{-}$, since $F(x, t) \geq 0$ by $\left(f_{5}\right)$, we obtain that

$$
\Phi(u)=-\frac{1}{2}\|u\|^{2}-\int_{\mathbb{R}^{N}} F(x, u) \mathrm{d} x \leq 0
$$

This, together with Lemmas 3.1 and 3.2, implies that there exist $R>r>0$ such that

$$
\inf _{N} \Phi>0 \geq \max _{\partial M} \Phi
$$

In view of Lemmas 3.3 and 3.4, $\Phi$ satisfies condition $(C)$. By Theorem 2.2 we have that $\Phi$ possesses at least one nontrivial critical point, which is the nontrivial solution of problem (1.1).

Proof of Theorem 1.3 Since $f$ is odd, $\Phi$ is an even functional. By Lemmas 3.3 and 3.4 we know that $\Phi$ satisfies condition $(C)$. To apply Theorem 2.3, it suffices to verify $\left(A_{1}\right)$ and $\left(\mathrm{A}_{2}\right)$.

Define $Y_{k}$ and $Z_{k}$ as in (2.6). Recall that $\lambda_{\ell}<0<\lambda_{\ell+1}$. If $k>\ell$, then we have $Z_{k} \subset E^{+}$. Define $\beta_{k}:=\sup _{u \in Z_{k}}|u|_{p}$. Therefore by (2.4) and (3.1) with $\varepsilon=1 / 4 \tau_{2}^{2}$ we have

$$
\begin{aligned}
\Phi(u) & \geq \frac{1}{2}\|u\|^{2}-\frac{1}{4 \tau_{2}^{2}}|u|_{2}^{2}-C|u|_{p}^{p} \\
& \geq \frac{1}{2}\|u\|^{2}-\frac{1}{4 \tau_{2}^{2}}|u|_{2}^{2}-C \beta_{k}^{p}\|u\|^{p} \geq \frac{1}{4}\|u\|^{2}-C \beta_{k}^{p}\|u\|^{p} .
\end{aligned}
$$


Let $r_{k}=\left(2 p C \beta_{k}^{p}\right)^{1 /(2-p)}$. Then for $u \in Z_{k}$ with $\|u\|=r_{k}$, we have

$$
\Phi(u) \geq \frac{1}{2}\left(\frac{1}{2}-\frac{1}{p}\right)\left(2 p C \beta_{k}^{p}\right)^{1 /(2-p)} .
$$

Since $\beta_{k} \rightarrow 0$ as $k \rightarrow \infty$ by [10, Lemma 3.8] and $p>2$, it follows that

$$
b_{k}=\inf _{u \in Z_{k},\|u\|=r_{k}} \Phi(u) \rightarrow \infty .
$$

Hence $\left(\mathrm{A}_{1}\right)$ is satisfied. Finally, by Lemma 3.2 with $W=\bigoplus_{j=0}^{k} \mathbb{R} e_{j}$ we easily see that $\left(\mathrm{A}_{2}\right)$ holds.

\section{Acknowledgements}

The authors would like to thank Professor Shibo Liu for reviewing an early draft of this paper and giving valuable comments

\section{Funding}

This work was supported by the National Natural Science Foundation of China (nos. 11701251, 11671185, 11771195), the Natural Science Foundation of Shandong Province (nos. ZR2017BA015, ZR2019YQ04).

\section{Abbreviations}

Not applicable.

\section{Availability of data and materials}

Not applicable.

\section{Competing interests}

The authors declare that they have no competing interests.

\section{Authors' contributions}

All authors contributed equally to this paper. All authors read and approved the final manuscript.

\section{Author details}

${ }^{1}$ School of Mathematics and Statistics, Linyi University, 276000 Linyi, China. ${ }^{2}$ College of Information Technology, The University of Suwon, 18323 Hwaseong-si, Republic of Korea.

\section{Publisher's Note}

Springer Nature remains neutral with regard to jurisdictional claims in published maps and institutional affiliations.

Received: 29 January 2021 Accepted: 21 April 2021 Published online: 01 May 2021

\section{References}

1. Kryszewski, W., Szulkin, A.: Generalized linking theorem with an application to a semilinear Schrödinger equation. Adv. Differ. Equ. 3(3), 441-472 (1998)

2. Bartsch, T., Liu, Z., Weth, T.: Sign changing solutions of superlinear Schrödinger equations. Commun. Partial Differ Equ. 29(1-2), 25-42 (2004). https://doi.org/10.1081/PDE-120028842

3. Liu, S.: On superlinear Schrödinger equations with periodic potential. Calc. Var. Partial Differ. Equ. 45(1-2), 1-9 (2012). https://doi.org/10.1007/s00526-011-0447-2

4. Jeanjean, L.: On the existence of bounded Palais-Smale sequences and application to a Landesman-Lazer-type problem set on $\mathbf{R}^{N}$. Proc. R. Soc. Edinb., Sect. A 129(4), 787-809 (1999). https://doi.org/10.1017/S0308210500013147

5. Coti Zelati, V., Rabinowitz, P.H.: Homoclinic type solutions for a semilinear elliptic PDE on $\mathbf{R}^{n}$. Commun. Pure Appl. Math. 45(10), 1217-1269 (1992). https://doi.org/10.1002/cpa.3160451002

6. Zeng, J., Li, Y.: Existence of solutions for an elliptic equation with indefinite weight. Nonlinear Anal. 66(11), 2512-2519 (2007). https://doi.org/10.1016/j.na.2006.03.034

7. Willem, M., Zou, W.: On a Schrödinger equation with periodic potential and spectrum point zero. Indiana Univ. Math. J. 52(1), 109-132 (2003). https://doi.org/10.1512/iumj.2003.52.2273

8. Bartsch, T., Ding, Y.: On a nonlinear Schrödinger equation with periodic potential. Math. Ann. 313(1), 15-37 (1999). https://doi.org/10.1007/s002080050248

9. Ding, Y., Lee, C.: Multiple solutions of Schrödinger equations with indefinite linear part and super or asymptotically linear terms. J. Differ. Equ. 222(1), 137-163 (2006). https://doi.org/10.1016/j.jde.2005.03.011

10. Willem, M.: Minimax Theorems. Progress in Nonlinear Differential Equations and Their Applications, vol. 24 , p. 162 Birkhäuser, Boston (1996). https://doi.org/10.1007/978-1-4612-4146-1

11. Jeanjean, L., Tanaka, K.: A positive solution for an asymptotically linear elliptic problem on $\mathbb{R}^{N}$ autonomous at infinity. ESAIM Control Optim. Calc. Var. 7, 597-614 (2002). https://doi.org/10.1051/cocv:2002068 
12. Li, Y., Wang, Z.-Q., Zeng, J.: Ground states of nonlinear Schrödinger equations with potentials. Ann. Inst. Henri Poincaré, Anal. Non Linéaire 23(6), 829-837 (2006). https://doi.org/10.1016/j.anihpc.2006.01.003

13. Szulkin, A., Weth, T.: Ground state solutions for some indefinite variational problems. J. Funct. Anal. 257(12), 3802-3822 (2009). https://doi.org/10.1016/j.jfa.2009.09.013

14. Reed, M., Simon, B.: Methods of Modern Mathematical Physics. II. Fourier Analysis, Self-Adjointness p. 361. Academic Press, New York (1975)

15. Reed, M., Simon, B.: Methods of Modern Mathematical Physics. IV. Analysis of Operators p. 396. Academic Press, New York (1978)

16. Rabinowitz, P.H.: On a class of nonlinear Schrödinger equations. Z. Angew. Math. Phys. 43(2), 270-291 (1992). https://doi.org/10.1007/BF00946631

17. Bartolo, P., Benci, V., Fortunato, D.: Abstract critical point theorems and applications to some nonlinear problems with "strong" resonance at infinity. Nonlinear Anal. 7(9), 981-1012 (1983). https://doi.org/10.1016/0362-546X(83)90115-3

18. Rabinowitz, P.H.: Minimax Methods in Critical Point Theory with Applications to Differential Equations. CBMS Regional Conference Series in Mathematics, vol. 65, p. 100. Am. Math. Soc., Providence (1986) Published for the Conference Board of the Mathematical Sciences, Washington, DC

19. Bartsch, T: Infinitely many solutions of a symmetric Dirichlet problem. Nonlinear Anal. 20(10), 1205-1216 (1993). https://doi.org/10.1016/0362-546X(93)90151-H

20. Liu, S.B., Li, S.J.: Infinitely many solutions for a superlinear elliptic equation. Acta Math. Sinica (Chin. Ser.) 46(4), 625-630 (2003)

\section{Submit your manuscript to a SpringerOpen ${ }^{\circ}$ journal and benefit from:}

- Convenient online submission

- Rigorous peer review

- Open access: articles freely available online

- High visibility within the field

- Retaining the copyright to your article

Submit your next manuscript at $\boldsymbol{\Delta}$ springeropen.com 